\title{
Multidimensional comparisons of structures of vocally and facially expressed emotion
}

\author{
REX S. GREEN and NORMAN CLIFF \\ University of Southern California, Los Angeles, California 90007
}

\begin{abstract}
The cognitive structure of vocally expressed emotion was examined using a nonmetric multidimensional scaling analysis and a factor analysis of seven tone-of-voice rating scales. A canonical correlation analysis suggested that the same two dimensions clearly emerged from these analyses, pleasant-unpleasant and excitement. These two general dimensions were each highly related to single tone-of-voice rating scales, those for pleasantness and thinness, respectively. The two dimensions of pleasant-unpleasant and excitement were shown to correspond to the facial expression scales of pleasant-unpleasant and attention-rejection developed by Engen, Levy, and Schlosberg in 1958. When the stimuli for both vocally and facially expressed emotion were mapped together on a two-dimensional space, notable differences became apparent. An attempt to interpret some of these differences was made.
\end{abstract}

People seem to be able to recognize emotional fluctuations in others by consistently noting certain nonverbal behaviors. Such consistency has led investigators to hypothesize that people have developed similar cognitive structures, or intemalized representations of these nonverbal behavior patterns, that makes the consistent determination and dillerentiation of various emotions possible (Cliff \& roung. 1968). The purpose of the present study was twololl: (1) to clarify the structure people have incernalized to help them recognize emotions expressed by tone of voice. and (2) to compare this structure to the structure used in the perception of lacially expressed emotion.

\section{The Structure of Emotion}

Most of the research on the perception of emotion has focused on the way people recognize emotion from facial cues. Pleasantness and activation are the two structural dimensions which consistently emerge as important determinants of people's perception of ficially expressed emotion.

Threce examples of studies of facial cues all found these same two dimensions to be the primary ones, though their methods of scale construction differed. Engen. Lery, and Schlosberg (1958) measured the facial expression of emotions by employing predetermined unidimensional scales, which they labeled pleasant-unpleasant. attention-rejection, and sleep-tension. Abelson and Sermat (1962) recovered two interpretable dimensions that corresponded most to the lirst and third of Engen et al.'s unidimensional scales. Their scaling model was a metric multidimensional one in which the jud ges determined the dimensions by making judgments of overall similatity. Cliff and Young (1968) obtained results

Rex S. Green's present address: Psychology Department, U.C.L.A., Los Angeles, California 90024. similat to these with a nonmetric multidimensional scaling model. In the nonmetric approach, only monotonic correspondence is required between the judgments of similarity and the interpoint distances used in the scaling model's representation of people's cognitive structures. Each of these three studies also found that the emotion stimuli formed a V-shaped pattern in these two dimensions. Both pleasant and unpleasant stimuli were high on the activation dinension, while stimuli low on activation were neither very pleasant nor unpleasant.

Numerous other studies reviewed by Dittman (1972) and Strongman (1973) provide further evidence that people have some consistent approach to identifying the emotions that others express facially. Two to four meaningful dimensions reoccur across studies. Beyond pleasantness and activation, there has been less agreement as to what the other two dimensions might be.

People also make judgments about their perception ol emotions based on cues other than facial ones. The tone of voice people use to express emotions, for example, maly also provide important clues which help other people to accurately perceive the emotion being expressed. However. there is little empirical knowledge about the internalized structure people use to judge emotions from tone-of-voice cues. The strongest assertion Strongman (1973) could make in his review was that adults can recognize emotion communicated by tone of voice better than chance and that. therefore, some emotion does seem to be conmunicalted in this way.

Argyle (1972) mentions tone of voice as one of many of the nonverbal aspects of speech. He notes that $\because$... |these aspects arel not closely linked with language. do not have a complex structure, and are similar to other expressions of attitudes and cmotions." Brooks (1971) and Ostwald (1963) provide more detailed categorizations of these nonverbal 
aspects of speech. Of the four mentioned by Brooks. the tones of voice or vocal qualifiers, such as pitch. loudness. and tempo seemed to provide important cues to a speaker's emotion.

Three empirical studies which attempted to represent the structure of rocalized emotion have encountered difficulties in finding a clear structure underlying the recognition of emotion from vocal cues. Davitz (1964) presented 14 emotions rocalized in the form of standard sentences as stimuli. He had subjects judge these. stimuli on nine semantic differential scales. The rank-order positions of some but not all of the emotions on those scales representing the pleasantness and activation dimensions coincided with the results of the three studies of facial expressions (Abelson \& Sermat. 1962: Cliff \& Young. 1968; Engen et al.. 1958). Smets (1967) replicated Davitz's study by gathering similarity judgments and using a metric multidimensional scaling analysis. In relating her study to Davitz's, the results were not very consistent across dimensions. Her attempt to relate the dimensions of judged vocalized emotion to independent acoustical measures did not clarify the structure much further. Dawes and Kramer (1966) took a fully nonmetric scaling approach to obtain a representation of the structure of vocalized emotion. Subjects identified vocalized emotions from a list. The extent to which they incorrectly identitied some emotions provided an index of the confusability of the emotions. A nonsymmetric matrix of confusions was analyzed for the distance information on five stimuli. The dimension recovered appeared to differentiate stimuli as to their perceived pleasantness. except that grief was judged more pleasant than indifference. Based on the existing research, it is clear that the cognitive structures of vocally expressed emotion have yet to be sufficiently determined.

Research to date which compares structures of the different modes of expressing emotion is limited to a study by Bradley (1969) of the verbal. facial, and postural modes of emotional expression. He obtained similarity judgments both across and within modes from the same subjects. Then he performed several nonmetric multidimensional scaling analyses in which he found that (1) the differences between modes were minimal compared to the high degree of correspondence. (2) the facial and verbal modes were most similar, and (3) the postural mode was about equally different from the other two. Bradley's comparisons were within the same study in which stimuli from different modes were presented together and the representation of the cognitive structure obtained in the same analysis. Other approaches to comparing modes of expression are needed such as comparisons across studies before much can be asserted about the relative similarities of the different modes.

\section{Some Methodological lssues}

All examination of the methedeleng lesed in the thee presiously mentioned studies of rocally expressed emotion revealed line problem atreas that presented better reconery of the structure of vocalized cmotion. The tirst three concern the construction of the stimuli. First cateh study used standard content sentences a present the tones of voice. It is rery likely that the werbal content whenes the emotions resealed by the sender, tone of wice. Alternatives to presenting verbal stimuli. such as reciting leflers of the alphabet or masking the verbal content mechanically. "sould reduce the verbal content. A second problem of previous researeh may have been that some of the other nomverbal aspects of speech besides the fone of voice received too much emphasis in the expression of some of the emotions. For instance, amusement may also be expressed in a litugh. which Broohs $\left(19^{-1}\right)$ catcgorized differently trom cones of roice. Furthermore. other aspects of speech. such as pauses. respiration rate, and speech rate. may unduly intluence the judgments of emotion when sentences are being judged. Greater control of these unwanted cues may be desirable. Third, in those studies where the different emotions are expressed by different senders. additional variance in the judgments is generated. At this point. it seems better (1) hold constant as many situational features as possible until a clear structure emerges. In addition to holding sender constant. the sender can be required to express each emotion in ternıs of a specitic situation (Engen et al.. 1958).

The last two problem areas concern the analyses of the data. Despite the fact that each of the studies of vocalized emotion employed different scaling methods, none of the three methods used seem best suited to studving rocalized emotion stimuli. The metric multidimensional scaling and semantic differential approaches require subjects to supply numbers at an interval-scale level of meaning. Perhaps subjects can only provide sufficiently consistent information at an ordinal scale level. The fully nonmetric approach employed by Dawes and Kramer (1966) was soundly conceived but lacked enough stimuli to determine even their one dimension clearly. Besides. it may be possible to recover a metric representation of people's cognitive structures which the fully nonmetric approach does not provide. Finally. it may be advisable to employ more than one method to analyze vocally expressed emotion. Structures obtained from two methods can be cross-checked to verify interpretations of the dimensions or patterns of stimuli. If a method in which subjects determine the dimensions is compared 10 one in which dimensions were preselected, the relative importance of the dimensions may be revealed by the former method while the latter approach will serve to more objectively identify the dimensions. 
One purpose of this study was thus to employ an improved methodology which would make it possible to determine a more clearly detined and interpretable structure underlying the recognition of vocally expressed emotion. Each of the five methodological problem areas was considered in the research design. First. insteald of standard content sentence stimuli, letters of the alphabet were used to express each cmotion. In this way, the extent of both the verbal content and several other nonverbal aspects of speech was minimized. Second, in the construction of these stimuli, aly effort was also made to minimize the use of cues other than tones of voice, such as chuckling, crying, screaming, etc. Third, situational determinants were pinpointed for each emotion and only one sender was used to construct the stimuli. In this waly. much undesired variance due to factors that were not of interest was eliminated. Fourth, a scaling model was selected that requires only ordinal information from subjects while yielding a metric representation of the underlying cognitive structure. $\Lambda$ larger stimulus set was constructed, as is required by this model, to sufficiently determine two dimensions. Finally, the multidimensional scaling analysis was supplemented by an analysis of a separate set of semantic differential scales representing various tones of voice. By comparing the two structures of vocally expressed emotion, it was hoped that the dimensions could be clearly determined.

A second purpose of this study was to compare the cognitive structure of vocally expressed emotion to that for facially expressed emotion. In the study by Bradley (1969) in which structural comparisons between modes of emotional expression were attempted, there were a number of methodological problems. Bradley's tindings of high consistency across modes was greatly enhanced by three features of his design. First, by making comparisons within the same study, some or all of the consistencies across judges, type of judgment, presentation of the stimuli, ctc., could account for part of the correspondence he found between modes. Second, by using only one scaling approach, he ruled out the possibility of finding less consistency across modes when measured by different models. Third, the small differences between the stimuli across modes, relative to the large differences among different emotions, tend to be eliminated by the nonmetric scaling algorithm he used. Since he scaled all the stimuli together, the differences across modes were minimized in obtaining the best fit of the recovered structure to the data.

In order to avoid these difficulties in making comparisons across modes of expression in the present study, the cognitive structure of vocally expressed emotion was compared to a previously obtained structure of facially expressed emotion (Engen et al., 1958). Their study was selected because it differed methodologically, in many ways, from the present study. The types of judgments obtained, the sex of the sender, the method of presentation, and the scaling methods, all differed. Thus, the possibility that some consistency in methods was accounting for the similarities between modes of expression was rendered unlikely. The problem of clustering stimuli that differ only by mode of expression was eliminated by performing separate analyses on each mode. The two structures were then rotated to maximize congruence so that the correspondence between modes could be examined.

In summary, this study was designed to clarify the structure of vocally expressed emotion and to determine the comparability of this structure to one obtained previously for the recognition of facially expressed emotion. The particular design of this study attemipts to overcome problems that may have hindered previous investigations on this topic.

\section{METHOD}

\section{Stimuli}

A pilot study using 21 bipolar scales of tones of voice culled from Brooks (1971) and Ostwald (1963) was conducted. Subjects judged 20 emotion words for each of the 21 scales. Principal components factor analyses were performed on both the scales and the emotions. Based on the factor analysis of the scales, seven of the bipolar scales were chosen. Two scales were chosen to represent each of the four factors whose eigenvalues were greater than one, except for the last dimension where only one scale was chosen. These scales in order of the dimensions recovered were: (a) hi-low pitch and sharp-flat, (b) warm-cold and pleasant-unpleasant, (c) disrupted-even and impulsive-reverberant, and (d) thick-thin.

Nine emotions were selected from a previous study of facial expressions (Engen et al., 1958) which could be represented clearly by tone of voice. Also, these emotions appeared distributed along the V-shaped pattern noted by Abelson and Sermat (1962), Cliff and Young (1968), and Engen et al. (1958), and they tended to be spread apart from one another. These nine emotions are listed in Table 1 along with two more described below. The numbers refer to corresponding facial expressions scaled by Engen et al. In order to have a stimulus set large enough for the MDS model, two more stimuli were selected from the vocalized emotion literature (Davitz, 1964; Feldstein, 1964). Normal conversational tone $(N)$ was selected to see if it would correspond in location to the facial stimulus of light sleep. Joy, upon receiving a desired present, was selected to balance the set of stimuli with equal numbers of pleasant and unpleasant stimuli.

A male undergraduate drama student was found who was willing to create the 11 stimuli. A tape recorder was set up in an experimental room, and the student was seated before the mike. The experimenter described the emotion and its related situation to him. The sex was changed for emotions 16 and 32, to make them appropriate for a male. The student was asked to speak using only letters of the alphabet while trying to emit the appropriate emotion. The student was instructed to put himself in the situation, to feel the emotion, and then on signal to start speaking letters with the emotion. The student did this for each emotion. After each emotion was recorded for $12 \mathrm{sec}$, the recording was played back. If either the experimenter or the student was not satisfied that the emotion was the one intended, another recording was made. In only two or three instances were second recordings required.

Eight-second sections were selected from each stimulus and rerecorded. Pairs of the 8 -sec stimuli were formed so that the order of the stimuli was randomly determined, as was the order of the 
Table 1

Emotion Stimuli Descriptions

\begin{tabular}{cl}
\hline Designator & Emotion Portrayed \\
\hline 7 & Grief-mother just died of cancer \\
12 & Disdain \\
15 & Very pleasant surprise (told he is going to get a lead \\
& part in a play) \\
16 & Paternal love-baby in arms \\
29 & Anger at seeing a dog beaten \\
32 & Unexpected meeting with old girl friend of whom \\
35 & he is still fond \\
36 & Response to an amusing story \\
51 & Sores \\
$\mathrm{J}$ & Joy uponledge that plane he is on will crash any moment \\
$\mathrm{N}$ & Normal conversational tone
\end{tabular}

pairs. For 11 stimuli, 55 pairs were required. Two tape recorders were set up to put the 8-sec stimuli into pairs. Four seconds separated the members of each pair. and $15 \mathrm{sec}$ separated pairs. Sixteen pairs were selected to be repeated and added after the 55 were recorded. Three trial pairs of stimuli were placed before the 55 on the tape, as were the instructions for the subjects telling them how to make the judgments.

\section{Subjects and Apparatus}

Seventeen subjects from introductory psychology courses participated in this experiment. Nine of the subjects were males and all subjects were $18-21$ years old. All subjects were placed in individual booths with earphones and listened to the tape of instructions and stimuli. The subjects recorded their judgments on computer-scored forms with No. 2 pencils.

\section{Procedure}

The subjects were instructed to listen to each pair, then mark a number from 1 to 9 to indicate the degree of similarity between the stimuli. where 9 represented least similar. After all these pairwise stimuli were presented, instructions were given telling the subjects how to make judgments on each of the seven bipolar adjective scales. A randomly determined order for the seven scales was reversed for every other stimulus in the test booklets. A random order for the 11 stimuli was also determined. Each stimulus was then presented by itself for $8 \mathrm{sec}$. The subjects were asked to rate it on up to four scales. It was presented again after $15 \mathrm{sec}$ had elapsed. The subjects were then requested to judge the stimulus on the remaining scales. Seventy-seven judgments were required in all from each subject for this part of the study. The total time required for the subjects to make all the judgments was about $1 \mathrm{~h}$ and $15 \mathrm{~min}$.

\section{Analyses}

Prior to averaging the judgments, a cluster analysis was run on the 17 sets of 55 judgments to determine if the subjects differed from one another in systematic ways. No distinct set of subgroups emerged. so only the average solution was interpreted. The similarity judgments were averaged across subjects to form one vector of 55 average judgments of the pairs of emotions. This vector was analyzed by TORSCA8 (Young \& Torgerson, 1967), a nonmetric multidimensional scaling algorithm. Solutions were obtained in one to five dimensions.

The judgments on the adjective scales were analyzed in a principal components factor analysis. Since both the MDS structure and adjective scale structure proved to be multidimensional, a canonical correlation analysis between the MDS structure and the seven scales was performed. This analysis yielded comparisons between the two structures of vocalized emotion.
The MDS solution, including only the nine stimuli which were comparable to those in the Engen et al. study, was matched with the three scales they used to obtain judgments of facial expressions. The loadings for facial expression were transformed to make them comparable to the vocal loadings in this study. Both sets of loadings were then analyzed by a factor matching algorithm developed by Cliff (1966). This algorithm positions the two structures so that the squared differences between corresponding stimuli are minimized while orthogonal axes or dimensions are retained. Direct comparisons of the two modes of expression were then made.

\section{RESULTS}

The five nonmetric multidimensional scaling solutions for one to five dimensions yielded stress values of $.21, .11, .05, .03$. and .02 . These were plotted to determine the optimal dimensionality. Larger stress typically occurs as fewer dimensions are employed to represent the configuration of points. Increasing the dimensionality of the solution to lower stress eventually produces very minimal improvement. The "elbow" in the stress values over the third dimension suggested that further dimensions yielded relatively smaller improvements in stress. Therefore, only the first three dimensions were retained for interpretation.

Further support for the existence of three MDS dimensions was given by the measures of predictive validity for each of the five scaling solutions. Distances between selected stimuli obtained from the MDS solutions were used to predict the corresponding repeated judgments given by the subjects in addition to the initial 55 judgments. Spearman's rho was calculated between the dissimilarity judgments and their corresponding distances for each solution, yielding five indices of predictive validity, .61, .67, $.86, .87$, and .87 . Using the criterion that a solution of greater dimensionality should increase predictive validity, the four-and tive-dimension solutions clearly failed to add sufficient predictive power.

The loadings for the three dimensions in their principal components position are presented in Table 2. The first dimension extended from normal conversational tone $(\mathrm{N})$ to fear of a plane crash (51). The pattern of loadings suggested that the first dimension be called "pleasant-unpleasant." Stimulus locations revealed that all the typically pleasant emotions had positive loadings except joy (J). Unpleasant emotions were represented by negative loadings, with the exception of revulsion (36). On the second dimension, the most widely separated stimuli were panic (51) and revulsion (36), emotions which differ in level of excitement, interest, or activity. The order of the remaining stimuli generally supported calling this second dimension "excitement." The origin of the dimension loadings in Table 2 was shifted to reflect this unipolar interpretation of the second dimension. The highest positive and negative loadings for the third dimension contrasted grief (7) 
Table 2

Dimension Loadings: Vocalized Emotion

\begin{tabular}{crrr}
\hline & \multicolumn{3}{c}{ Dimensions } \\
\cline { 2 - 4 } Stimulus & 1 & \multicolumn{1}{c}{2} & \multicolumn{1}{c}{3} \\
\hline 7 & -.64 & .52 & .43 \\
12 & -.24 & .40 & -.41 \\
15 & .26 & 1.00 & .29 \\
16 & .12 & .48 & -.06 \\
29 & -.43 & .78 & -.32 \\
32 & .46 & 1.16 & -.04 \\
35 & .67 & .94 & -.22 \\
36 & .05 & .13 & .07 \\
51 & -.69 & 1.32 & -.04 \\
J & -.36 & .80 & .17 \\
$\mathrm{~N}$ & .79 & .62 & .12 \\
\hline
\end{tabular}

Note-Origin translated to approximate the base of the $V$.

and disdain (12). Intense grief is associated with crying uncontrollably, yielding to the pressure of events. Disdain is an expression of superiority over events as though to resist then. Tentatively, the third dimension was identified as yielding-resisting. Further support for this interpretation was inferred from the loadings associating surprise (15) and joy (J) with yielding and anger (29) with resisting. Four stimuli had near-zero loadings on this dimension. implying that these emotions were not particularly associated with this continuum.

Although the nonmetric multidimensional scaling analysis provided a sufticiently meaningful threedimensional representation for all 11 stimuli, some unexpected tindings emerged. Normal tone was expected to be emotionally neutral, but its pattern of loadings indicated that possibly two of the three dimensions are needed to describe it. Another finding $w$ as that $J$ was located among the negative emotions. This unexpected deviation was the only clear contradiction to the dimensional interpretation of the vocal MDS structure.

The arrangement of the points representing the locations of the stimuli in the three-dimensional space were then compared to the cone-shaped arrangement of facially expressed stimuli hypothesized by Engen et al. Starting with the first two dimensions that are depicted in Figure 1, a V-shaped structure did appear to underlie the locations of the nine emotions originally studied by Engen et al. A cone-shaped arrangement in three dimensions would require the stimuli with larger loadings, such as 51 and 32 , to have higher loadings on the third dimension, while 36 should have a near-zero loading. Since all three had near-sero loadings, a cone-shaped structure did not seem appropriate to describe the relationships between the emotions.

An examination of the interpoint distances was made to determine if a tetrahedron would enclose these stimuli, the four vertices corresponding to four outlying stimuli. Bradley (1969) found such a representation to be adequate for happy, sad, angry, and fearful in his cross-modal study. Outlying stimuli best representing these emotions were selected out of the 11:35. 7, 29, and 51, respectively. A structure somewhat resembling a tetrahedron emerged. However, the vertex represented by anger (29) was too close to the other stimuli, and amusement (35) was too far away from the negative stimuli. Also, two points lay well beyond this structure, 36 and $\mathrm{N}$, and 12 was somewhat outside the tetrahedron. Since the $V$-shaped two-dimensional structure found in the studies of facial expressions included 36 , it was added as an outlying point to represent the bottom of the two-dimensional $V$-shaped configuration. These five vertices described a four-sided pyramid. The base of the pyramid was roughly square-shaped and consisted of the negative emotions. Amusement (35) was represented by the peak of the pyramid, and 12 was located outside this structure somewhat nearer anger than revulsion.

In order to examine the underlying factors for the seven adjective scales representing tones of voice, a principal components analysis was performed. The tirst two principal components had eigenvalues of 5.7 and 1.9. Since the other eigenvalues were negligible, the tirst two components were varimax-rotated and interpreted (see Table 3). As all the scales were bipolar, negative correlations implied that a reflection of the scale would yield a similar positive correlation. The first factor was very highly related to the thin-thick and high-low pitch scales. That is, this factor was most characteristic of both the pitch of one's voice and its thinness of sound. The second factor was highly related to the pleasant-unpleasant and warm-cold scales, so that tones of voice sounding pleasant and warmer to unpleasant and colder were represented by this factor.

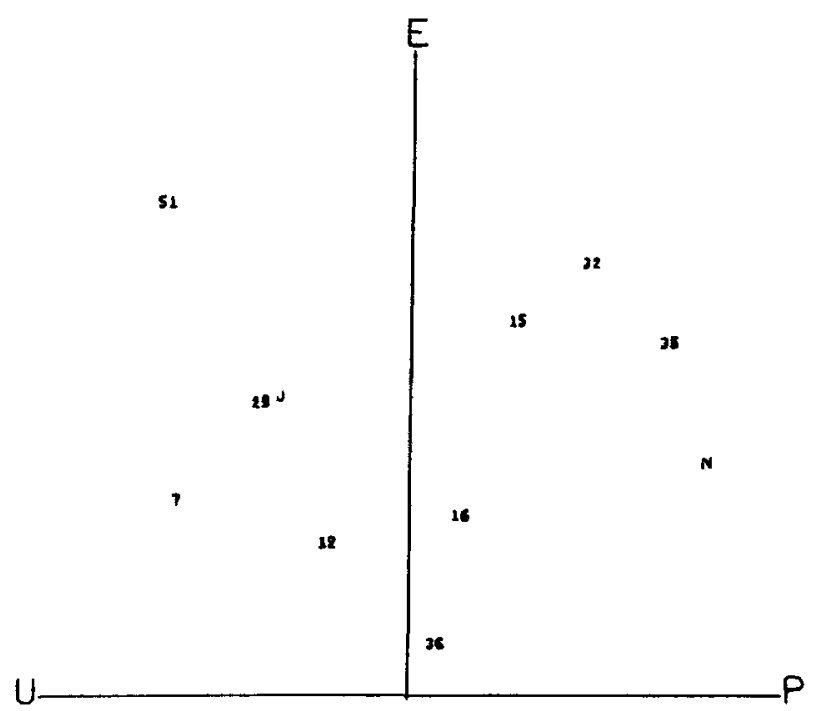

Figure 1. First two dimensions of vocalized emotion. 
The two vocal structures were then compared by a canonical correlation analysis. Canonical variates for the three MDS dimensions were correlated with canonical variates for the seven tone-of-voice scales. All of the possible canonical correlations were signiticant: .999 and .984 at $p<.01$ and .959 at $\mathrm{p}<.05$. Clearly, the two vocal structures were highly related. Another means of demonstrating the degree of relationship between structures is through the redundancy of their variances (Cooley \& Lohnes, 1971). Across all three pairs of canonical variates, the redundancy or the proportion of variance of the three dimensions accounted for by the seven scales was $95 \%$, while the redundancy in the opposite direction was $83 \%$.

An initial attempt to interpret the pattern of regression coefficients for the three pairs of canonical variates was unsuccessful. Following Mulaik (1972), factor loadings of the variables on the two sets of canonical factors were calculated. Clarification of the factors from the pattern of factor loadings was sought through an orthogonal hand rotation. The second two canonical factors for the tone-of-voice scales were aligned so they would best match the most nearly orthogonal scales, warm-cold and thin-thick. During rotation the two sets of canonical factors were constrained to maintain the overall level of their relationship. The resulting pattern of factor loadings of the variables on their respective sets of canonical factors are depicted in Table 4. Along with the loadings, the redundancies for the dimensions, $R_{m}$ (proportion of variance accounted for by the scales), and those for the tone-of-voice scales. $\mathrm{Rd}_{\mathrm{s}}$, are reported for each pair of canonical factors.

The extent to which the canonical factors corresponded to the two targeted scales, warm-cold and thin-thick, is indicated by the very high loadings on the second factor for warm-cold and on the third factor for thin-thick along with near-zero loadings on other factors. The pattern of loadings for the tone-of-voice scales on the second and third canonical factors was very similar to the previously determined varimax-rotated factor loadings. The order of the factors is reversed, however. The pattern of redundancies indicated that for the first pair of canonical factors the MDS dimensions were redundant to the tone-of-voice scales, but that the

Table 3

Factor Loadings of Rating Scales

\begin{tabular}{lcc}
\hline Scale & Factor I & Factor II \\
\hline Pleasant-Unpleasant & -.20 & -.95 \\
Warm-Cold & .03 & -.98 \\
High-Low Pitch & .98 & .12 \\
Sharp-Flat & .92 & .35 \\
Thin-Thick & .95 & -.21 \\
Impulsive-Reverberant & .72 & .64 \\
Disrupted-Even & .75 & .54 \\
\hline
\end{tabular}

Table 4 Canonical Factor Loadings

\begin{tabular}{lrrr}
\hline & \multicolumn{3}{c}{ Canonical Factors } \\
\cline { 2 - 4 } & $\mathrm{I}$ & II & III \\
\hline MDS Dimensions & & & \\
I & -.33 & .81 & -.49 \\
2 & -.48 & .31 & .82 \\
3 & .83 & .47 & .31 \\
Rating Scales & & & \\
P-U & -.34 & .90 & -.16 \\
W-C & -.07 & .98 & .06 \\
H-L & .08 & -.19 & .97 \\
S-F & -.07 & -.37 & .91 \\
T-T & .04 & .05 & .91 \\
I-R & .05 & -.62 & .66 \\
D-E & .04 & -.34 & .74 \\
Redundancy & & & .31 \\
Rdm & .33 & .31 & .47 \\
Rd & .02 & .34 & \\
\hline
\end{tabular}

variance in the scales was not accounted for by the dimensions. Examining the pattern of loadings, the relationship of the third dimension to the seven scales was not simple. The pattern of factor loadings for the second two sets of canonical factors suggested that the first and second dimensions were more simply related to certain of the tone-of-voice scales.

A direct comparison of the MDS dimensions with the tone-of-voice scales was made by finding the correlations of the dimensions with the canonical factors for the scales (Cooley \& Lohnes, 1971). All the variables were then plotted on the second and third canonical factors for the scales. From Figure 2, it is apparent that the canonical factors correspond well with the two target scales, warm-cold and thin-thick. The pleasant-unpleasant dimension extended well out in the canonical factor space and pointed in a direction similar to the pleasant-unpleasant scale. Thus, the first MDS dimension was most highly related to the second canonical factor along with the warm-cold and pleasant-unpleasant scales. The second dimension and the thin-thick scale were similarly aligned with the third canonical factor. The high-low pitch scale was also highly related to this factor. The third dimension did not extend very far from the origin and was not located near either of these canonical factors or near any of the tone-of-voice scales.

The alignment of the first dimension and the pleasant-unpleasant tone-of-voice scale strongly supported the identification of the first dimension of vocalized emotion as pleasant-unpleasant. The first dimension appeared to be highly related to the warmth of expression, also. The second dimension, excitement, appeared to be characterized most by increases in the thinness of voice and in vocal pitch.

Plots of the other two pairs of canonical factors were examined, but no tone-of-voice scale proved to extend far enough in a similar direction to the third 
MDS dimension to reveal which tones of voice are most characteristic of this dimension. The pattern of correlations on the first pair of canonical factors along with the small $\mathrm{Rd}_{\mathrm{s}}$ discouraged any further analyses.

The MDS dimensions were not rotationally determinate in this analysis. The positions of the tirst two dimensions in the canonical factor space suggested that they could be rotated about $25 \mathrm{deg}$ to better match the warm-cold and thin-thick scales. If they were so rotated, they would suggest directions in the MDS space corresponding more to those two tones of voice.

With the meaningfulness of the MDS structure of vocalized emotion established for the first two dimensions along with a tentative description for the third dimension, this vocal structure was then compared to the structure of facial expressions of emotion determined by Engen et al. (1958). They gathered judgments on three scales, pleasantunpleasant, attention-rejection, and sleep-tension, to identify facially expressed emotion. The scale values for the nine emotional stimuli, taken from their data and used in the present study, were extracted from Morgan (1971) and transformed to be comparable to the MDS rocal loadings. Using Case II of Cliff's (1966) factor matching procedure, the MDS loadings were rotated to congruence with the facial expression scales by al least-squares procedure. The pattern of correlations indicated that the first MDS dimension corresponded highly to Engen et al.'s pleasantunpleasant scale, that the second MDS dimension was highly related to the attention-rejection scale and somewhat related to the sleep-tension scale, and that the third MDS dimension was somewhat related to

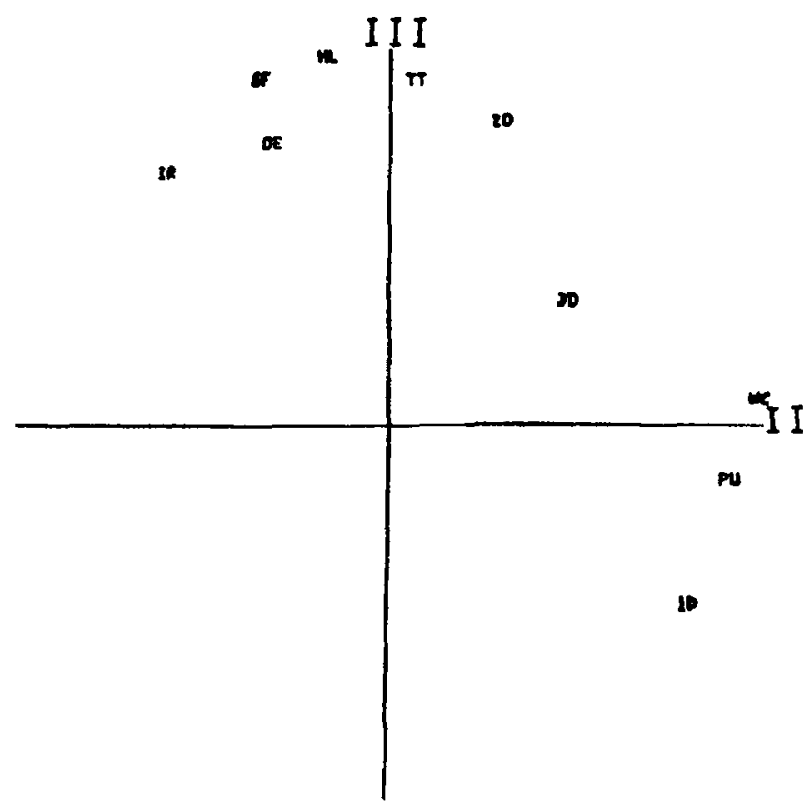

Figure 2. Canonical factors for the tone-of-voice rating scales.

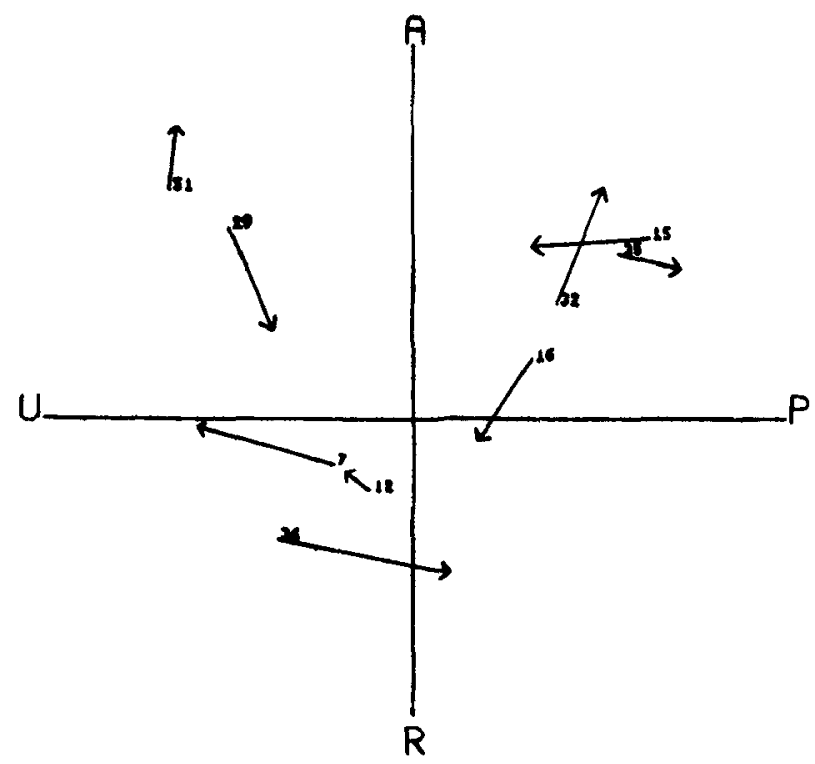

Figure 3. Facial-to-vocal comparisons on the first two facial scales.

both the attention-rejection and sleep-tension scales. Unfortunately, the discovery that the second and third facial expression scales were highly related $(r=.85)$ across the nine stimuli made it impossible to identify all three MDS dimensions of vocalized emotion in this analysis. However, no rotation at all was needed to match the first two MDS dimensions in their principal components position to the first two facial expression scales, the correlations being .85 and .89 . The first two MDS principal components were taken to match the pleasant-unpleasant and attention-rejection scales, respectively.

In order to examine the discrepancies between the facial and vocal stimuli, the loadings of the vocal stimuli were transformed with reference to the pleasant-unpleasant and attention-rejection facial expression scales. Both sets of stimuli were plotted together using the facial expression scales as axes (see Figure 3). The arrowheads denote the vocalized stimuli. The greatest discrepancy occurred between the facial and vocal expressions of revulsion (36). As the arrow indicates, the vocalization was judged more pleasant than the facial expression. On the other hand, grief (7) sounded more unpleasant than its facial expression. In general, it appeared that the meaningfulness of the two structures was quite similar but that some of the stimuli differed noticeably from one mode of expression to the other. Figure 3 clearly shows that the two-dimensional $V$ shape, which approximately described the facial expression stimuli relations, also describes the vocal stimuli. Since the pattern of discrepancies was mixed with arrows of varying lengths pointing in many different directions. it seemed unlikely that some consistent difference distinguished the two modes of expression. 


\section{DISCUSSION}

Despite difficulties encountered in previous attempts to obtain a clear and meaningful structure of vocalized emotion, the results of this study amply demonstrated that such a structure can be recovered. Evidence of meaningfulness for two dimensions of vocalized emotion was gathered from all three analyses performed. In addition, meaningful structural relationships among the emotional stimuli were found in the MDS and factor matching analyses.

The MDS structure was interpreted in terms of three dimensions: pleasant-unpleasant, excitement, and yielding-resisting. The locations of nearly all the emotional stimuli supported these interpretations. The alignment of the first dimension in the canonical factor space with the tone-of-voice scale, pleasantunpleasant, confirmed this interpretation of the first dimension. The second dimension was aligned with two tone-of-voice scales which were construed to depict level of excitement in vocal expressions, thin-thick and high-low pitch. The third MDS dimension was not closely aligned with any tone-of-voice scale, leaving its interpretation unconfirmed by the canonical correlation analysis. The variance in the MDS dimensions was very well accounted for by the two canonical factors which nearly matched the warm-cold and thin-thick tone-of-voice scales. From Figure 2 emerged the possibility that an alternative interpretation of the MDS space could be made by orthogonally rotating the first and second MDS dimensions about $25 \mathrm{deg}$ towards these two scales. The first dimension would then approximate the warmth of expression and the second dimension the thinness of voice.

In the factor matching analysis, the match of the first two vocal dimensions to two scales of facial expressions studied previously lent further support to the present dimensionsal interpretation of the vocal structure. The pleasant-unpleasant vocal dimension matched the facial expression scale of the same name. The vocal dimension of excitement matched the attention-rejection scale for facial expression. Recent reviews of the literature have pointed out the importance of the pleasantness and activation dimensions in the recognition of facially expressed emotion (Dittman, 1972; Strongman, 1973). This study demonstrated that vocal expression of emotion is described by two dimensions similar in meaning to those for facial expressions and that the order of importance of the two dimensions is the same.

Beyond the finding of meaningful dimensions in the vocal expression of emotion, the structural relationships among the stimuli proved meaningful. The MDS analysis showed that the emotion stimuli, with the exception of $N$, were organized in a $V$-shaped pattern in the first two dimensions. The factor matching analysis showed that this $\mathrm{V}$-shaped pattern was similarly oriented to the $\mathrm{V}$-shaped structure previously found for the facial expression stimuli. Greater pleasantness or unpleasantness was associated with greater excitement, while low levels of excitement accompanied expressions of minimal pleasantness or unpleasantness.

Although the two $\mathrm{V}$-shaped structures appeared in similar orientations, there were sizable shifts across modes for some of the stimuli. These shifts were not surprising, since there were numerous methodological differences between the two studies and since they were conducted 15 years apart. A pattern in the shifts would have indicated a notable difference between the two modes. However, since the shifts occurred in all directions and with varying degrees of discrepancy between modes, the implication was that the shifts were random or related specifically to each emotion. Some contradiction was provided to the interpretation of the facial scales by the shifts in 16 and 36 from the facial to vocal modes. The vocal expression of love (16) moved onto the rejection side of the second facial scale and revulsion (36) crossed over to the pleasant side of the pleasant-unpleasant scale. Across all the shifts in locations of the emotion stimuli, no pattern emerged that indicated that the discrepancies were more meaningful than the similarities in the interpretations of the two structures. Bradley's (1969) findings were essentially upheld by this factor matching analysis.

\section{Areas for Further Exploration}

The results of this study raise a number of questions for further consideration. First, has the complete structure of vocally expressed emotion been recovered? All three analyses, MDS, canonical correiation, and factor matching, provided consistent agreement about the meaning of the first two dimensions. However, the interpretation of the third dimension identified in the MDS analysis as yielding-resisting was neither confirmed nor disconfirmed in the two subsequent analyses. The factor spaces for both the seven tone-of-voice scales and the three facial expression scales were essentially two-dimensional. None of the individual tone-of-voice scales appeared near the third dimension in the space of canonical factors. Furthermore, the linear combination of tone-of-voice scales most highly related to the third dimension bore no interpretable structure. Also, the finding that the second and third facial scales were highly related to each other precluded matching all three of the MDS dimensions to the three facial scales. Thus, no further identification of this dimension could be made from comparisons with the unidimensional scales, vocal or facial. Since these other measurement procedures did not confirm the interpretation of the third MDS dimension, the nature of the vocal structure remains uncertain beyond two dimensions. Perhaps even a 
fourth dimension exists but could be recovered only if a wider variety of emotions expressed vocally were scalled.

Although the structure of vocalized emotion closely resembled a four-sided pyramid, the structure was only roughly defined. The base of the pyramid was not quite square because the vertex representing anger did not appear far enough from the other negative emotions. Disdain and anger seemed to be located along two edges of the square that intersected beyond their locations. If a more extreme emotion such as rage had been included, the square-based pyramid might have been formed more clearly.

If a pyramidal structure represents the complete structure of vocalized emotion, verification of this structure relative to other possibilities requires scaling many more stimuli than 11 . Some stimuli might be included as test stimuli which are expected to lie outside the structure of vocalized emotion. Notably, $\mathrm{N}$ was an outlier relative to the pyramid structure in three dimensions. Being a vocalization, it may be described in terms of the dimensions of vocalized emotion, yet it might not belong in the structure of emotion. The pattern of interstimulus distances for $\mathrm{N}$ with the other vertices did not suggest any particular form as an alternative to the pyramid structure. It seemed most likely that $\mathrm{N}$ was not related in some systematic way to the other stimuli. A complete structure of emotion should clearly indicate what its boundaries are, perhaps revealing which stimuli are not emotions as well as which are. The continuing use of appropriate multidimensional scaling models along with comparisons to other structural interpretations of facial expressions (Bradley, 1969; Engen et al., 1958; Frijda, 1968; Osgood, 1966) should aid in determining a more complete structural representation of vocalized emotion.

Second, can vocally expressed emotions be adequately generated in the laboratory as stimuli for research? Joy (J) was located within the pyramid structure but was inappropriately located on the first MDS dimension. It was also judged as less pleasant or warm than the other pleasant emotions on the tone-of-voice scales. In the three-dimensional structure, it was positioned midway between 29 and 7 . Replays of the pairings of $J$ with 29 and 7 confirmed the high degree of similarity of $J$ to these two negative emotions. The similarity of $J$ to these two emotions may be due to the fact that positive feelings associated with joy were poorly represented by the sender in the construction of this stimulus. Since his representations of the other stimuli were apparently quite good, some further reason for the poorer representation of this stimulus was sought. If vocal cues for joy are more subtle than smiling, more extensive experiencing of joy may be required of the sender before he can appropriately reconstruct this emotion vocally. Schutz (1967) claimed that many people lack such experience with joy. This may make joy more difficult to express vocally.

An examination of the discrepancies between the facial and vocal stimuli indicated some further problems that may have been present in the construction of the stimuli. Some relatively large shifts in point locations from facial to vocal modes for the emotion stimuli occurred for $7,15,16,29,32$, and 36. The discrepancies for $7,16,29$, and 32 were not of interest for interpretation, however, since excessive error was present in fitting the MDS model to their data. This was determined by calculating the stress values for each stimulus. The stress for each of these four stimuli proved to be greater than the stress across all stimuli. Only the shifts in 15 and 36 were not associated with individual point stress greater than the stress for all distances. The location of 15 as more pleasant facially than vocally may have been due to the fact that pleasantness, represented by the smile in the facial expression, was not as easily represented in the vocal mode. Lacking a simpler cue, such as a smile, the vocalization of 15 perhaps did not contain cues which would be perceived as sufficiently pleasant. The implication of these conjectures is that construction of emotional stimuli in a laboratory may fail to capture the most appropriate representation of each emotion.

Difficult as vocalizations are to properly reconstruct in the laboratory, most of the emotions were represented well enough to recover a very meaningful structure of vocalized emotion. For example, the other large discrepancy for Stimulus 36 did not appear to be due to a problem in constructing the stimulus, but rather the discrepancy may have resulted from the fact that two different judgment procedures were used. In both of the unidimensional scaling tasks, facial and vocal, 36 was rated as unpleasant on the pleasant-unpleasant scale, regardless of mode. It appeared that the discrepancy found between modes was also a discrepancy between judgment tasks. The pairwise comparison method employed in the MDS analysis resulted in locating 36 improperly on the pleasant side of the first dimension. Certainly there are difficulties in representing emotions vocally in a laboratory setting. By performing parallel analyses as in the present study, some of the deficiencies were discovered and a meaningful recovery of the structure of vocalized emotion was still realized.

In summary, the particular research design and scaling procedures employed in the present study successfully answered the questions posed by this research, but raised new questions and problems as well. The design produced affirmative results to the research questions of: "Can a meaningful structure of vocalized emotion be recovered?" and "Are different modes of emotional expression perceived similarly?" This research raised questions about the completeness 
of the structure of vocalized emotion that was recovered and about the problem of adequately representing emotional stimuli in laboratory research. Clearly, the advantages of combining MDS with factor analysis in a research design was demonstrated. The fact that there was a high correspondence of MDS dimensions to specitied unidimensional scales suggests the usefulness of the MDS approach for future research in a number of areas, such as preferences, attitudes, and clinical judgments.

\section{REFERENCES}

Abelson, R., \& Sermat, V. Multidimensional scaling of facial expressions. Joumal of Experimental Psychology, 1962, 63, 546-554.

ARgYle, M. Non-verbal communication in human social interaction. In R. A. Hinde (Ed.), Non-verbal communication. Cambridge: University Press, 1972.

Bradley. P. A multidimensional scaling of mood expressions. Unpublished doctoral dissertation, University of Southern California, 1969.

Brooks, W. D. Speech communication. Dubuque, lowa: Brown. 1971.

Cliff. N. Orthogonal rotation to congruence. Psychometrika, $1966,31,33-42$.

Cliff, N., \& Young, F. On the relation between unidimensional judgments and multidimensional scaling. Organizational Behavior and Human Performance, 1968, 3, 269-285.

COOLEY, W. W., \& LoHnes, P. R. Multivariate data analysis. New York: Wiley, 1971.

DAvitz, J. Auditory correlates of vocal expressions of emotional meanings. In J. Davitz (Ed.), The communication of emotional meaning. New York: McGraw-Hill, 1964.
DAWES, R. M., \& KRAMER, E. A proximity analysis of vocally expressed emotion. Perceptual and Motor Skills, 1966, 22, 571-574.

Dittman, A. L. Interpersonal messages of emotion. New York: Springer, 1972.

Engen, T., Levy, N., \& Schlosberg, H. The dimensional analysis of a new series of facial expressions. Journal of Experimental Psychology. 1958. 55, 454-458.

Feldstein, $S$. Vocal patterning of emotional expression. In J. Masserman (Ed.), Science and psychoanalysis (Vol. 8). New York: Grune \& Stratton, 1964.

Frijda, N. H. Recognition of emotion. In L. Berkowitz (Ed.). Advances in experimental social psychology (Vol. 4). New York: Academic Press, 1968.

Morgan, C. T. Study guide for introduction to psychology. New York: McGraw-Hill, 1971.

Mula1k, S. The foundations of factor analysis. New York: McGraw-Hill, 1972.

Osgood, C. E. Dimensionality of the semantic space for communication via tacial expressions. Scandinavian Journal of Psychology, 1966, 7, 1-30.

Ostwald, P. Soundmaking: The acoustic communication of emotion. Springfield, Ill: Thomas, 1963.

Schutz, W. C. Joy. New York: Grove Press, 1967.

SMETs, G. Multidimensional scaling of vocal emotional expressions with standard verbal content. Psychologica Belgica, 1967, 7. 75-85.

Strongman, K. T. The psychology of emotion. London: Wiley, 1973.

Young, F., \& Torgerson, W. S. TORSCA, a FORTRAN IV program for Shepard-Kruskal multidimensional scaling analysis. Behavioral Science, 1967, 12, 498.

(Received for publication February 1, 1974; revision received January 7,1975 .) 